at first quiet and then violent, the delirium time after time passing into a renewed convulsion and repetition of events; and this may go on for hours, or even days, in spite of any other treatment, and yet yield at once to Indian hemp. Further, with regard to genuine epilepsy and allied affections, I have found this, that Indian hemp has been, on the one hand, useful only in those cases where the diagnosis has led me away from a belief in the presence of the really classical disease, to the suspicion of organic lesion or of eccentric irritation; and, on the other hand, entirely useless, when there is no doubt of the nature of the malady. In many cases of genuine epilepsy, where attacks of petit mal are exclusively present and very frequent, and have been so for years, I have given Indian hemp in gradually increasing doses until some slight toxic effeet is produced; but have never found it to reduce either the frequency or severity of the seizures, or to materially affect their character. In tonic spasm, such as torticollis and the like, in writer's cramp, in meneral chorea, in paralysis agitans, in the jerking movements of spinal sclerosis, in trismus, and in tetanus, Indian hemp has proved in my experience absolutely useless ; but at the same time it is a most valuable medicine in the nocturnal cramps of old and gouty people; it in some cases relieves spasmodic asthma, and is of great service in cases of simple spasmodic dysmenorrhoca.

Second, in explaining the occasional toxic effects of Indian hemp, two things nuust be remembered:-

1. That the drug is one which, by its nature and the forms of its administration, is liable to great variations in strength. For practical purposes, its active principle has not been separated, and extracts, as well as tinctures made from the extract or from the plant, cannot be made uniform ; because the hemp grown during different seasons, and in different places, varies in the amount that it contains of the therapeutic agent. It is desirable, therefore, that it should always be obtained from the same source, and that a minimum dose should be given to begin with, and that the close should be very gradually and cautiously increased.

2. That individuals differ widely in their relations to many medicines and articles of diet, and pethaps to none more widely than to those of vegetable origin - such as tea, coffee, ipecacuanha, dizitalis, nux ronuca, and the like; and therefore, in addition to securing purity of drug, the possibility of idiosyncrasy should be borne in mind, to emphasise the need of caution, in the first administration of Indian hemp. By habituation and gradual increase of the dose, two, three, or even four grains of the extract nay be taken not only with impunity, but with advantage; but such a dose as one grain wonld, so far as my experience goes, be attended with toxic effects in the majority of healthy adults. I have seen them in mild form from one-third of a grain, and very rarely from one-fourth, but have never known them arise from one-tifth. Therefore, for an adnlt 1 always give onefifth or less to commence with, and for a child one-tenth. The best, because most convenient, form for administration is the tincture. Pills often become hard and insoluble, and their strength cannot be so readily and so gradually in creasel. The tincture, if suspended by mucilage, is apt to separate in the mixtmre that contains it, and thus the doses become uneven. Therefore, during many years I have given it with instruetions that the dose required should be taken in drops on a small piece of sugar or bread. The tincture of the Pharmacopoeia contains one grain in about twenty minims, and this is convenient for use with children; but for the adult, where a gradually in creasing dose is required, a tincture with a strength of one grain in ten drops is more useful. The dose should be given in minimum quantity, repeated in not less than four or six hours, and gradually increased by one drop every third or fourth day, until either relief is obtained, or the drug is proved, in such case, to be useless. With these precautions I have never met with any toxic effects, and have rarely failed to find, after a comparatively short time, either the value or the uselessness of the drug

It is no surprise to me that "W. W." should have suffered as he did, and which he so accurately and graphically describes, from the dose that he had taken; nor does it astonish me that his friend, possessed of a different idiosyncrasy, could have taken it with impunity; and the object of this communication will be attained if, by giving my experience of the great value of Indian hemp, my brethren may be deterred from abandoning its use by any dread of its causing " toxic effects," unless it be given in a " toxic" dose.

\section{RETROVERSION OF THE GRAVID UTERUS; DEATH FROM RUPTURE OF THE BLADDER AND PERITONITIS.}

\section{By THOMAS OLIVER, M.A., M.D., M.R.C.P.,} PHYSICIAN TO THE ROYAL INFIRMARY, NEWCASTLE-UPON-TYNE.

I VENTURE to lay the following notes before a section of the readers of THE LANCET, believing that they will prove of interest to many young practitioners whose attention may not have been directed to a mode of death which occasionally supervenes in the course of pregnancy. The case is one to. which I was called in consultation by a medical friend a few miles from Newcastle. The patient died about two hours before I reached the farmhouse which she and her husband had occupied.

Mrs. C. H-, aged thirty-six, a delicate woman and the mother of four children, took ill on the 27 th of June, her complaint being vomiting accompanied by pain in the abdomen. She was seen during the course of the day by he: medical attendant, to whom was given the history of a five months' pregnancy, and by whom the ordinary medicines. used in these cases to allay sickness were ordered. On the following day her condition was worse; the patient looked ill, the vomiting and pain in the abdomen had not been relieved, the abdomen was distended more than the assertes period of pregnancy accounted for, and it was more generally tympanitic. The diagnosis was peritonitis complicating pregnancy; and although the illness was regarded as grave by her medical attendant, it did not suggest itself to hinn as one likely to terminate fatally on the following day. The 29 th of June found her in a condition worse than ever. For the first time he now learned that nn urine had bees passed for more than twenty-four hours. Thinking that the pain in the abdomen might be associated with the processes. of labour he made a vaginal examination, but failed to find? the os uteri. The patient was now in is state of collapse, and was evidently dying from peritonitis, the cause of which was obscure.

Althongh the patient was dead when I reached the house, the doctor was very anxious that I should make a vagina examination, in order to appreciate the difficulty he had experienced in trying to reach the "os." Cadaveric rigidity. had already set in. There was considerable difficulty to the passage of the finger into the vagina, owing to the fact that the posterior wall was nearly driven out of the vulva, the cause of the displacement being a soft cyst. lying behind it. The finger gliding in in front of this passed at once up behind the pubis, but no "os" could be felt. It was quite clear that the cyst.like body was part of $a$ displaced pregnant uterus, the " $\mathrm{os"}$ " of which had been lifted upwards and carried high above the pubis. Consent to a pust-mortem examination was readily obtained, and this we made on the following day.

On opening the abdomen there escaped a ouantity of thick brownish fluid, which smelt strongly of putrefying urine. About a pint and a half of this fluid was afterwards found in the abdominal cavity. When the walls of the abdomen were reflected, there was brought into view a bluish-red surface extending from the pubis up to the leve of the umbilicus. It looked at first as if it was the oute surface of a distended pregnant uterus. It was at places firmly adherent to the abdominal parietes and intestines. This turned out to be the outer surface of an elongated, Hattened, and ulcerated bladder, from which, by enlarging one of its perforations, I removed several ounces of the thick brown foul-smelling urine already described. Posteriorly the elongated and flattened bladder was firmly adherent to a large cyst-like body, the size of a child's head, which, passing downwards and backwards, occupied nearly the whole of the pelvis, and, continuing downwards into the vagina, had thrust before it the posterior wall of that passage. With considerable difficulty I raised this, and found it to be an enlarged pregnant uterus containing a five months' foetus. How, then, are we to account for all this? The bladder had towards its fundus formed adhesions with the intestines and abdominal walls. On cutting into it, its walls were found to be extremely thick at some places, and remarkably thin at others; especially was this the case towards the fundus anteriorly, where there was an area about an inch and a half square, extremely attenuated, perforated, and sloughing. The intestines were generally 
hyperæmic, and numerous flakes of lymph lay upon them. The liver was healthy, as were also the other organs of the abdomen. The cause of death was evidently perforation of the bladder and peritonitis. What part did the retroverted uterus play? The prominent symptoms were abdominal pain and vomiting, with retention of urine ; and if we had only the post-mortem appearances to guide us, it would be difficult to say whether these were the result of the uterine displacement, or whether the first two of these symptoms had depended upon peritonitis, and the frequent vomiting, with its attendant straining, had induced the displacement of the gravid uterus.

We got the following history. Less than a month prior to this Mrs. C. H- had been out walking some distance from home. All at once, she said, she "felt something drop in her inside." She walked a few steps further, but was obliged to sit down and wait until a conveyance was brought to take her home. For a few days she was confined to bed, and attended by a neighbouring practitioner. She recovered so far as to be able to resume her household duties, but only to have a return of pain and vomiting. Not improving under treatment, she exchanged her medical attendant for the one by whom I was called to the case. He therefore only saw her for the first time within three days of her death, and he tells me that at that tine there was no retention of urine, but a constant desire to micturate. A small quantity of urine only was being passed, and that with very great pain.

Denman attributed retroversion of the gravid uterus to distension of the bladder. Barnes and Playfair are not inclined to accept this as the usual explanation, nor do I see how such a cause unaided could act. Severe straining or injury operating at a time when the bladder was inordinately distended might induce displacement. Seeing how frequently displacement of the non-pregnant uterus occurs, it is astonishing how very seldom obstetricians meet with retroversion of the gravid uterus. In most of these cases pregnancy has arisen in the retroflexed organ, and the tendency is, as the pregnancy advances, for the uterus to right itself, although now and then the fundus doubtless gets caught by the promontory of the sacrum. Retroversion in such would therefore be in existence from the first, but no symptoms to any extent would be experienced until the uterus grew too large for the pelvis and began to press upon the bladder and rectum. Then would arise such symptoms as pain, retention of urine, tenesmus, and vomiting. Now that is scarcely the history of our case. We cannot overlook in the history of the case the walk, the sudden and sharp pain, the feeling as if something had all at once dropped in her insidesymptoms which made further progression a matter of impossibility to her, and which are more in keeping with a sudden displacement, such as prolapse of a pregnant uterus or a retroversion of it. Such an event occurring at a time when the bladder was distended would be associated with a backward and downward direction of the fundus, and the weight of the intestines bearing upon the anterior surface of the womb would roll the fundus towards the sacrum and at the same time raise the os upwards and forwards behind the pubis. Once this is accomplished the straining which vomiting causes would tend to wedge the uterus still further downwards and outwards. In our case it would appear as if these had aggravated the displacement. The uterus had descended and had been driven downwards with considerable force, dragging and pressing upon the biadder, which, already adherent and thinned at places, had become perforated, leading to extravasation of urine, collapse, peritonitis, and death. I am inclined to think, therefore, that there was a considerable element of suddenness in the retroversion of the gravid uterus in this case, and from the difficulty which I experienced in trying to lift up the retroverted organ with my hands in the abdominal cavity, I can appreciate the severity of the task which would have been undertaken by any obstetrician in his attempt to have replaced it by the vagina during the life of the patient. Reduction of the bulk of the uterus by withdrawal of liquor amnii through the " os" if it can be reached, or by puncture through the uterine wall, is a procedure that may have to be undertaken by those who are unfortunate enough to meet with such a case. Before resorting to this, however, emptying the bladder by means of the catheter should be tried, for such simple treatment combined with rest in bed has been followed time after time, not only by a disappearance of serious symptoms, but by a rectification of the displacement.

Newcastle-upon-Tyne.

\section{TROPICAL LIFE AXD ITS SEQUEL $A$.}

BY SURGEON.GENERAL JEFFERY A. MARSTON, M.D., C.B , M.R.C.P., \&c.

(Concluded fiom p. 5\%0.)

MORE or less anamia, like debility, occurs, of course, after every ordinary, or every serious or protracted illness, but it is more observable in hot climate, because in the case of a febrile attack, for example, the deteriorating effects of the fever heat have been supplemented by those of climatic heat. Anicmia embraces a wide range of degree. It is not necessary to dwell upon those simple forms which occupy a sort of borderland between health and disease; nor is it my intention to speak of the anæmia which may be reasonably expected to ensue in consequence of previous sickness, or of that which can be accounced for by the presence of some organic or other disease; but rather of those varieties in which anæmia seems to be the disease-cases in which it may be regarded as primary and not secondary in character. The number of individuals that appear in the course of a few years before medical boards who are suffering from different varieties and degrees of anæmia is considerable. There is no very clear practical line of demarcation between the simpler and graver forms-that variety of essential anæmia, for instance, which is progressive and renerally fatal. The graver kinds of climatic anæmia, those which in some degree mimic the pernicious variety, differ from the latter in some important respects. In regard to age (the sutferer from climatic or tropical anæmia may be of any age); he differs in not having the peculiar yellow, waxy, cachectic appearance; in the absence of retinal homorrhages; and in his recovering, instead of the disease progressing to a fatal issue as in the pernicious form. The sufferers from climatic anæmia are often young men; they do not, as a rule, exhibit signs of much wasting, but they are often very debilitated; there may or may not (generally not) be signs of structural heart disease. The motions are usually natural in colour; the urine, though of low specific gravity, is rather high coloured than otherwise, and the amount of urea is relatively large, which would point to increased hamolysis. The anxmia is not so much due to a diminution of quantity as deterioration of quality of the blood. I have no observations, however, as to the extent to which the red corpuscles had decreased, nor any regarding an increased quantity of iron in the hepatic tissue. As to the pathology of this climatic anæmia, whether it has any points of identity or close alliance with that of pernicious anæemia or not, $I$ have no special information.

It is astonishing how anæmic some individuals become after residence in hot climates, without having actually had any disease to account for it. They may or may not have suffered from intermittent fever, but they have commonly been exposed to malarious influences, although, as a rule, there is no marked splenic or hepatic enlargement. Some of these cases exhibit slight febrile disturbances-that is to say, the evening temperature reaches $100^{\circ}$.

There is one factor always present in the medical history of the cases under review; they have all been exposed to the effects of climatic heat. It is very difficult, if not im. possible, to apportion the influence of the separate causes or conditions present; but the patient need not necessarily have been exposed to malaria. Even where climatic heat seems to have been the cause of the anxmia, it is difficult to say how much was directly attributable to heat per se, and how much to the constitutional depression, increased perspiration, diminished appetite, and depressed digestive and nervous functions caused by it.

An officer of about forty-five years of age, after having spent some years in the plains of India, and enjoyed excellent health, went to Simla. At the latter station he began to lose strength, weight, and colour without any appreciable disease. There was no history of his having suffered from ague, still, I suspected that a malarious cause was at the root of his symptoms. After a time he noticed that he became subject to an attack of vomiting daily in the morning whilst his stomach was empty ; the vomited matter consisted entirely of a small quantity of bile, never of food. There was no fever, the tongue was clean, the appetite good; physical examination elicited nothing; his bowels were regular, but rather loose; urine acid, non-albuminous, free of 